\title{
EFFECT OF OIL CONTAINING n-3 POLYUNSATURATED FATTY ACIDS (PUFA) ON THE IMMUNE RESPONSE AND GROWTH FACTORS IN PIGLETS
}

\author{
R. KAŠTEL', Viera ReVAJOVÁ, D. MAGIC, J. Pistl, M. LEVKUT, L'. BINDAS, J. ŠAJBIDOR \\ and M. HORVÁTH
}

University of Veterinary Medicine, 04181 Košice, Komenského 73, Slovak Republic

(Received October 27, 1998; accepted March 1, 1999)

\begin{abstract}
Oral administration of n-3 polyunsaturated fatty acids (PUFA) to piglets slightly enhances the immune response. As compared to the control, in the experimental piglets the absolute values of monocytes in the peripheral blood were significantly increased $(\mathrm{P}<0.05)$, while the metabolic activity of phagocytes and the number of lymphocytes within the individual subpopulations were slightly higher. The level of growth factors, determined on the basis of somatomedin in the blood serum, was significantly higher in the experimental group $(\mathrm{P}<0.05)$. $\mathrm{n}-3$ PUFA interfere with the synthesis of prostaglandins and influence the metabolism of fatty acids. This finding may contribute to the therapy of inflammatory processes influencing immune and growth factors in piglets.
\end{abstract}

Key words: Polyunsaturated fatty acids, supplementation, lymphocytes, phagocytes, piglets

Recently, great attention has been paid to essential polyunsaturated fatty acids (PUFA) and their effect on the nutrition of piglets. An elevated intake of n3 PUFA decreases the level of triacylglycerides, very low-density lipoproteins (VLDL), cholesterol and low-density lipoproteins (LDL) in the blood plasma of experimental animals. The representation of eicosapentaenoic acid (EPA) and docosahexaenoic acid (DHA) at the expense of arachidonic acid (AA) is increased in plasma lipids (Herold and Kinsella, 1986). In some stages, there was an increase in HDL (high-density lipoprotein) cholesterol, while the effect of DHA was more pronounced than that of EPA (Morisaki et al., 1983; Nestel, 1986). The mechanism of n-3 PUFA action on plasma lipids has not been described yet. n-3 PUFA are expected either to decrease the rate of apoprotein B synthesis (Nestel et al., 1984) or the formation of VLDL triacylglycerols (Harris et al., 1984). n-3 PUFA decrease the synthesis of prostaglandins and shift the balance to a reduction in thrombocyte aggregation. Prostaglandins and leukotrienes also significantly influence immune functions and inflammatory reac-

"E-mail: kastel@uvm.sk; Fax: 00421956323666 
tions. n-3 PUFA use the lipoxygenase pathway, whereby mediators with reduced inflammatory activity are formed; by contrast, n-6 PUFA are metabolised by cyclo-oxygenase, and pro-inflammatory metabolites arise (Vaughn et al., 1994). Essential PUFA, acting as the structural components of cellular membranes and as precursors for eicosanoid production, are important modulators of humoral (i.e. they control saccharide metabolism) and cell-mediated immune reactions. In part this demonstrates that the effect of dietary PUFA on immune reactions, through their ability to metabolise, leads to eicosanoid production. Potential effects of PUFA on biological receptors, signal transduction and lymphocyte proliferation depend on the PUFA composition of immune cells.

The aim of this study was to find out whether n-3 PUFA were incorporated in the cellular membranes of immune cells in piglets. Their effects on the expression of cellular receptors as well as on the functional activity of lymphocytes and phagocytes in the peripheral blood were also studied. By analysing their concentrations in the blood serum, the production of growth factors and eicosanoids, as well as their effects exerted on the cells of the immune system of piglets were investigated.

\section{Materials and methods}

\section{Animals and diet}

The experiments were carried out on eleven, clinically healthy 4-day-old piglets (Landrace $\times$ Slovak White Improved crossbreds) weighing $1.2-2.0 \mathrm{~kg}$. The piglets were divided into two groups, with five piglets in the control and six in the experimental group.

During a 35-day period after birth, the six experimental piglets were orally administered the oil (Seal oil, fi Star Enterprises, Saint John, Newfoundland, Canada) containing essential PUFA, in a dose of $100 \mathrm{mg} \mathrm{kg}^{-1}$ body weight per day. The piglets were weighed every week, and the dose of oil was increased in proportion to their weight gain, i.e. from $0.4 \mathrm{ml}$ to $2 \mathrm{ml}$. Vitamin E ( $250 \mathrm{mg}$ ) was administered together with the oil. During the last week, piglets were additionally fed with ČOS 1, a commercial feed mixture (Agrocass, Košice, Slovak Republic).

\section{Blood sampling}

Three blood samples were withdrawn from the retroorbital venous sinus of the piglets' eye after each week of dietary treatment.

\section{Chemical composition of oil}

The chemical composition of oil containing an increased amount of $n-3$ PUFA is illustrated in Table 1. 
Table 1

Chemical composition of oil containing an increased amount of $n-3$ polyunsaturated fatty acids

\begin{tabular}{ll}
\hline \multicolumn{2}{c}{5 gram of oil includes } \\
\hline Total n-6 PUFA & $0.1 \mathrm{~g}$ \\
Total n-3 PUFA & $1.0 \mathrm{~g}$ \\
Total monounsaturated fatty acids & $2.6 \mathrm{~g}$ \\
Total saturated fatty acids & $0.9 \mathrm{~g}$ \\
Cholesterol & $0.005 \mathrm{~g}$ \\
\hline
\end{tabular}

\section{Immunological tests}

Assays for the analysis of immunological parameters were chosen to evaluate the functional activity of phagocytic cells and lymphocytes.

(a) Iodo-nitro-tetrazolium reductase test (INT). Quantitative evaluation of the tetrazolium reductase activity of phagocytes was carried out according to the method of Lokaj and Oburkova (1975) to determine the metabolic activity (MA) of phagocytes during phagocytosis. The leukocyte suspension $\left(1 \times 10^{7} \mathrm{ml}^{-1}\right)$ was divided into two parts and incubated at $37{ }^{\circ} \mathrm{C}$ for $45 \mathrm{~min}$. One portion was incubated with $1 \%$ starch suspension (Amylum oryzae) in phosphate-buffered saline (PBS) and the other without starch. All cellular suspensions contained INT (3/4iodophenyl-2-/4-nitrophenyl/-5-phenyl/-tetrazolium chloride, Lachema, Brno, Czech Republic). After the incubation and lysis of cells by acetone, the formasan content was determined by spectrophotometry at $485 \mathrm{~mm}$. The results are described in the form of an index of metabolic activity (IMA) based on the ratio of the mean optical density of leukocyte suspensions with starch $(\mathrm{n}=3)$ and leukocyte suspensions without starch $(\mathrm{n}=3)$.

(b) Leukocyte migration-inhibition assay (LMIA). The LMIA was used to analyse the reactivity of lymphocytes to mitogenic activation and was carried out according to Bendixen et al. (1976). The leukocyte suspensions were tested in a concentration of $2 \times 10^{8} \mathrm{ml}^{-1}$ under agarose with $5 \mu \mathrm{g} \mathrm{ml}{ }^{-1}$ mitogen (phytohaemagglutinin, PHA, Sigma, Germany) and without mitogen. The plastic dishes contained $5 \mathrm{ml}$ of $1 \%$ agarose consisting of culture medium (RPMI 1640, 10\% fetal calf serum, $100 \mu \mathrm{g} \mathrm{ml}^{-1}$ streptomycin and $100 \mathrm{IU} \mathrm{ml}^{-1}$ penicillin). After preincubation $\left(37^{\circ} \mathrm{C}, 1 \mathrm{~h}, 5 \% \mathrm{CO}_{2}\right)$, cell suspensions were transferred into holes in the agarose medium and incubated under the same conditions for $20 \mathrm{~h}$. The migration index (MI) was determined as a ratio of the mean of migration areas of leukocytes with and without mitogen. 
Flow cytometry

Primary monoclonal antibodies. Monoclonal antibodies (MoAbs) CD2, CD4 and CD8 were kindly provided by Dr. Trebichavský (Institute of Microbiology, Czech Academy of Science, Prague, Czech Republic) and SWC3 and IgM by Dr. Haversson (University of Bristol, Great Britain). The monoclonal antibodies used are summarised in Table 2.

Table 2

Monoclonal antibodies used

\begin{tabular}{llll}
\hline \multicolumn{1}{c}{ Specificity } & MoAbs & Isotype & Diluted \\
\hline CD2 & MSA4 & IgG2a & $1: 25$ \\
CD4 & $74-12-4$ & IgG2b & $1: 25$ \\
CD8 & $76-2-11$ & IgG2a & $1: 25$ \\
SWC 3 & $74-22-15$ & IgG1 & $1: 25$ \\
IgM $(\mu$ chain $)$ & K139.3E1 & IgG2a & $1: 25$ \\
\hline
\end{tabular}

Secondary antibody. Fluorescein isothiocyanate (FITC) conjugated sheep anti-mouse IgG (whole molecule) was used in a dilution of 1:128 (Immunochemicals, Sigma, Germany).

Isolation of lymphocytes. Venous blood samples were collected from all animals and put into ethylenediamine tetra-acetic acid (EDTA). Lymphocytes were separated by Ficoll-Hypaque gradient sedimentation (Boyum, 1974).

Procedure for flow cytometry (FACS). After the separation, the lymphocytes were washed twice with PBS supplemented with 0.2 per cent sodium azide and once in immunofluorescent medium (RPMI 1640 supplemented with 5 per cent fetal calf serum and 0.2 per cent sodium azide). Fifty $\mu 1$ of cellular suspension $\left(1 \times 10^{6}\right.$ lymphocytes in immunofluorescent medium) and $50 \mu 1$ of specific or control MoAbs were mixed and incubated at $4{ }^{\circ} \mathrm{C}$ for $30 \mathrm{~min}$. After incubation the cells were washed twice in the immunofluorescent medium (IFM) and pellets were mixed with $25 \mu 1$ of FITC-conjugated anti-mouse immunoglobulins and incubated as described above, in the dark. The working dilution of monoclonal and FITC-conjugated anti-mouse immunoglobulins was determined by titration and the calculation of a resolution index. After being stained the cells were washed twice in the IFM and once in PBS supplemented with 0.2 per cent sodium azide. The cells were resuspended in $0.5 \mathrm{ml}$ of the same buffer.

Analysis of stained cells. The FACS system (Becton Dickinson, Germany) was provided with $15 \mathrm{~mW}$ argon ion laser. The analysis examined a dot plot of the lymphocytes obtained by the forward and side scattering physical character of the lymphocyte population. The results are therefore expressed as the per- 
centage of the lymphocyte population, which was positive for a specific MoAb. The absolute numbers of lymphocytes were calculated by differential counts of leukocytes. An unrelated MoAb of similar isotype was used as control.

\section{Lipid extraction and analysis}

Lipids were extracted from the serum and transformed into methyl esters of fatty acids by the method of transesterification in methyl alcohol in an alkaline environment (Kaštel' et al., 1992). A Hewlett-Packard gas chromatograph, model 5890 A with 30-m capillary column (Supelcowax 10, Supelco Bellefonte, PA) was used for the determination of fatty acids of methyl esters.

Growth factor analysis

Somatomedin $\mathrm{C}\left(\mathrm{IgF}_{1}\right)$ was measured in blood serum by radioimmunoassay.

\section{Statistical evaluation}

Unpaired T-tailed test was used for statistical analysis.

\section{Results}

The evaluation of polyclonal activation of lymphocytes by mitogen revealed no differences in the mean values of the migration index (MI) in LMIA between groups of piglets. The MI was always within the range of the reference values (MI < 0.8, Fig. 1).

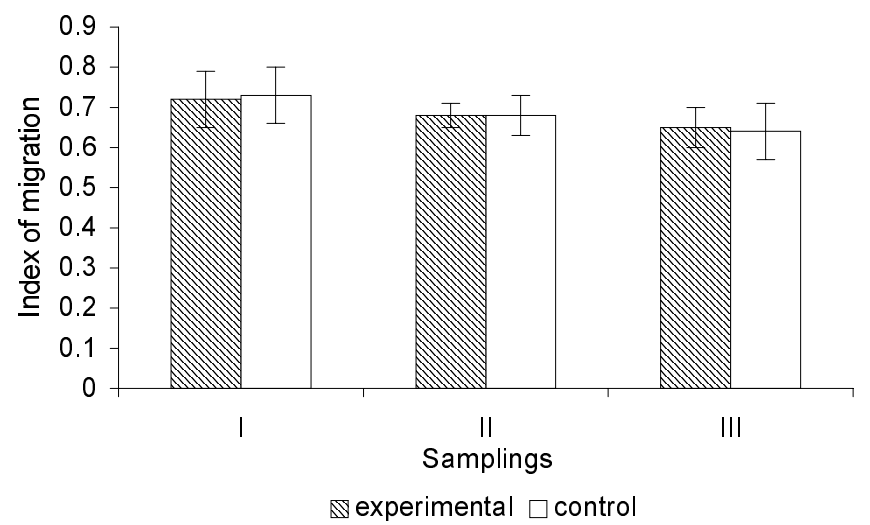

Fig. 1. The index of migration determined by leukocyte migration-inhibition assay to lymphocyte activation by phytohaemagglutinin. Bars indicate mean values while the lines represent SD 
At each sampling the index of metabolic activity (IMA) of phagocytes, indicating the enzymatic activity of phagocytes at load, was within the physiological limits in all the animals. In comparison with the controls, a slight increase in IMA was recorded in the experimental animals, which indicates a more intensive phagocytic activity (Fig. 2).

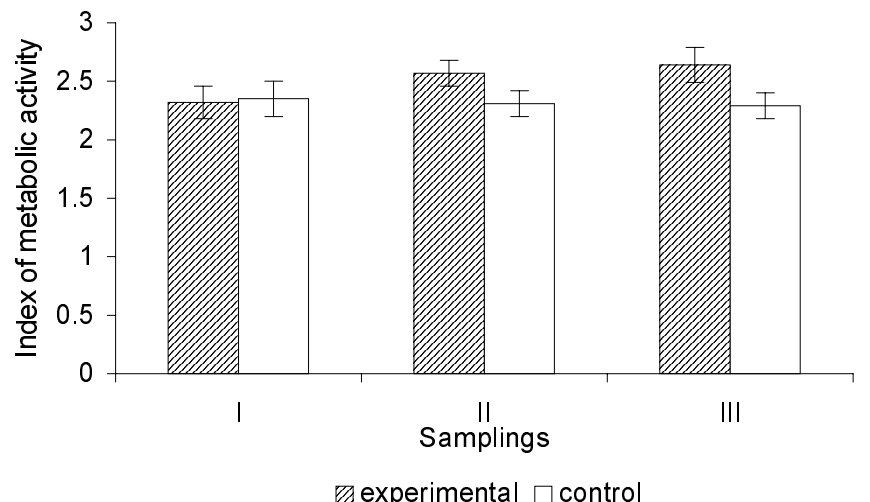

Fig. 2. The index of metabolic activity of phagocytes determined by iodo-nitro-tetrazolium reductase test. Mean values $\pm \mathrm{SD}$

The number of leukocytes in the peripheral blood was higher in the experimental animals than in the control ones at all the three samplings; however, the difference was not statistically significant. Moreover, the absolute numbers of lymphocytes harmonised well with the elevated values of leukocytes (Table 3 ).

Table 3

Mean values of leukocytes and absolute numbers of lymphocytes

\begin{tabular}{lrrrrr}
\hline & \multicolumn{2}{c}{$\begin{array}{c}\text { Leukocytes }\left(\mathrm{G} \mathrm{l}^{-1}\right) \\
\pm \mathrm{SD}\end{array}$} & & \multicolumn{2}{c}{$\begin{array}{c}\text { Lymphocytes }\left(\mathrm{G}^{-1}\right) \\
\pm \mathrm{SD}\end{array}$} \\
\cline { 2 - 3 } \cline { 5 - 6 } (weeks) & experimental & control & & experimental & control \\
\hline I (2) & $8.35 \pm 2.22$ & $7.12 \pm 1.80$ & & $5.60 \pm 1.38$ & $4.81 \pm 1.22$ \\
II (4) & $12.73 \pm 4.04$ & $9.10 \pm 2.53$ & & $8.76 \pm 3.80$ & $5.67 \pm 2.13$ \\
III (6) & $12.13 \pm 1.94$ & $11.46 \pm 1.17$ & & $6.71 \pm 1.74$ & $6.09 \pm 1.10$ \\
\hline
\end{tabular}

$\mathrm{SD}=$ standard deviation

The CD2-positive subpopulations of lymphocytes, largely $\mathrm{T}$ lymphocytes, contained higher numbers of cells in the experimental animals, but the difference was not significant. The determination of CD4-positive cells, characterising the 
population of helper or inductor lymphocytes, revealed an insignificant increase in the experimental group at sampling I $(1.70 \pm 0.39)$ as compared to the control $(1.40 \pm 0.32)$ and at sampling II $(2.96 \pm 1.14$ and $2.23 \pm 0.94$, respectively). The CD8-positive cell subpopulation, the so-called cytotoxic and suppressor lymphocytes, showed increased values in the experimental animals, even though the statistical significance of the difference was of a low level. The B lymphocyte subpopulation, measured by the expression of IgM molecules, indicated increased values in the experimental animals at sampling II $(1.17 \pm 0.38)$ as compared to the controls $(0.82 \pm 0.32)$, although the significance of the difference was low $(\mathrm{P}<0.09)$. At sampling II a significant increase was recorded in the measurement of the subpopulation positive for monocyte expression. This difference was significant at the level of $\mathrm{P}<0.05$ and was represented by values of $0.88 \pm 0.39$ and $0.55 \pm 0.22$ in the experimental and control group of piglets, respectively (Fig. 3).

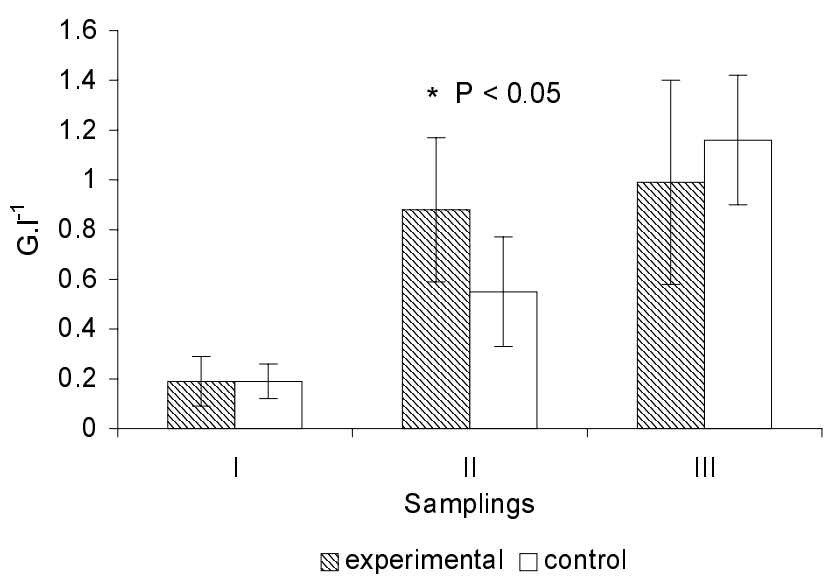

Fig. 3. Absolute number of monocytes determined by flow cytometry and calculated by differential count of leukocytes. Mean values $\pm \mathrm{SD}$

The growth factors detected in the blood serum of piglets by RIA using somatomedin measurement indicate a significant increase in the experimental group, where the levels of somatomedin ranged from $310.8 \pm 54.4 \mathrm{ng} / \mathrm{ml}$ at sampling II to $461.5 \pm 41.57 \mathrm{ng} / \mathrm{ml}$ at sampling III as compared to the control, where the level was $240.6 \pm 46.2 \mathrm{ng} / \mathrm{ml}$ and $332.8 \pm 42.14 \mathrm{ng} / \mathrm{ml}$ at sampling II and sampling III, respectively ( $\mathrm{P}<0.05$; Fig. 4$)$. 


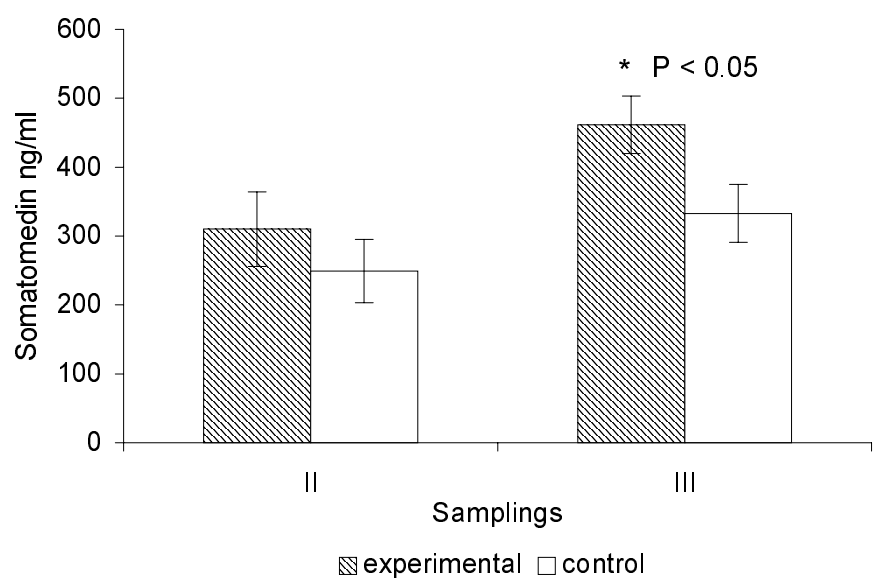

Fig. 4. Level of growth factor (somatomedin) determined in the blood plasma by RIA. Mean values $\pm \mathrm{SD}$

The administration of n-3 PUFA has been found to increase not only the level of gamma-linolenic acid (GLA), but also that of EPA and DHA (Fig. 5) at the expense of AA, the level of which decreased by one-fifth in comparison to the control. The values of DHA in the blood of piglets were significantly higher $(\mathrm{P}<0.05)$ in the experimental group $(1.8 \pm 0.39 \mathrm{~g} / 100 \mathrm{~g})$ than in the control group $(1.09 \pm 0.31 \mathrm{~g} / 100 \mathrm{~g})$. The level of arachidonic acid was significantly increased $(\mathrm{P}<0.05)$ in the experimental group $(7.05 \pm 0.27 \mathrm{G} / 100 \mathrm{~g})$ as compared to the control group $(4.7 \pm 0.18)$.

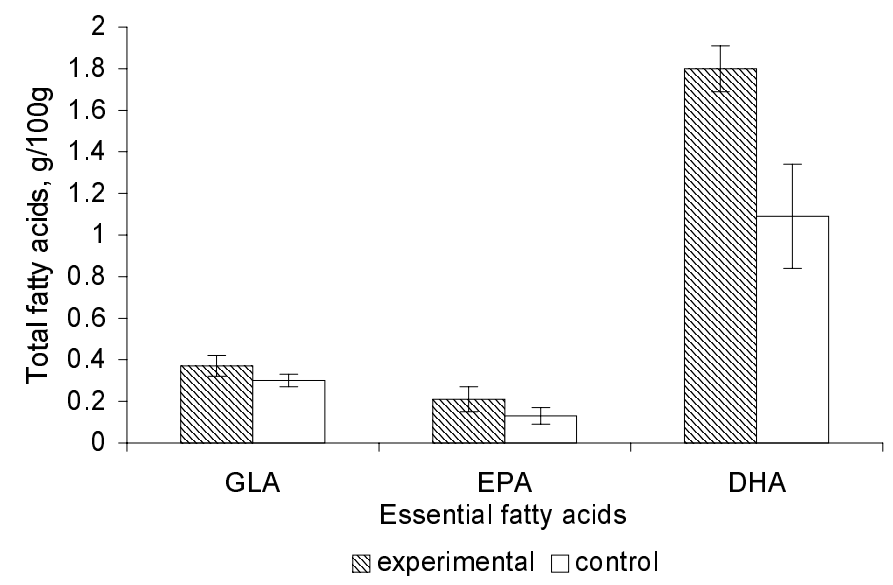

Fig. 5. The level of fatty acids GLA (gamma-linolenic acid), EPA (eicosapentaenoic acid) and DHA (docosahexaenoic acid) in blood lipids after administration of essential fatty acids. Mean values $\pm \mathrm{SD}$ 


\section{Discussion}

Enrichment of immune cells with n-3 fatty acids has been demonstrated in rats, mice, chickens and humans when n-3 PUFA were provided directly in the diet of the experimental subject (Fritsche et al., 1993a).

The present study demonstrates a moderate effect of n-3 PUFA containing diet on the immune response of piglets. The measurement of lymphocyte activation by mitogen did not reveal any differences in the mean values of MI; this indicates that the response of lymphocytes to a mitogen stimulus was not influenced by $n-3$ PUFA, or this test is not sufficiently sensitive. In comparison with the control group, a slight increase in IMA was found in the experimental group at samplings II and III; this may indicate a more intensive phagocytic activity. A significant increase $(\mathrm{P}<0.05)$ at sampling II was also recorded in the subpopulation positive for monocyte expression. The growth factor levels also increased significantly.

The most substantial changes were found in the fatty acid composition of the blood with respect to EPA, DHA and AA, which are important precursors of some essential compounds. The AA value decreased 1.5 times in experimental piglets compared to the controls. This leads to a reduced production of prostaglandins, in agreement with other data of the literature (Fritsche et al., 1991; Fritsche et al., 1993b). EPA and other n-3 PUFA extrude AA from membranous phospholipids and so they influence lipid metabolism. This has been described in some species of mammals such as humans, cats, and rodents, first of all in rats and hamsters (German et al., 1996). The above-mentioned n-3 PUFA pass the lipoxygenase pathway while mediators with reduced inflammatory activity are formed. EPA has the same chemical structure as AA with the exception of one more double bond of n-3.

AA and EPA compete for cyclo-oxygenase. If lymphocytes contain an increased amount of EPA, they will produce smaller aggregation-active thromboxanes, TXA2, and replace them with TXA3 thromboxanes having a weaker aggregating effect. In the intestinal endothelium, prostacyclin PGI 3 arises from EPA, which has an anti-aggregating effect like PGI 2 arising from AA. Here, monocytes are transformed to macrophages that accumulate lipids from circulating lipoproteins and simultaneously release growth factors (somatomedins). Therefore, it is possible that an increased supply of n-3 PUFA can decrease the binding of monocytes to the endothelium, and in this way it acts preventively against atherogenesis (Lee et al., 1985).

The above findings indicate that oral administration of n-3 PUFA to piglets elevates the level of n-3 PUFA in the blood serum and has an effect on eicosanoid production. A slight increase of the immune parameters and a significant influence on the levels of growth factors and PUFA metabolism can be observed. Of the fatty acids, the level of GLA, EPA and DHA increased and that of AA decreased in piglets fed n-3 PUFA. These changes may contribute to the therapy of inflammatory processes. 


\section{References}

Bendixen, G., Bentzen, K., Clausen, J. E., Kjaer, M. and Saborg, M. (1976): Inhibition of human leucocyte migration. In: Natvig, J. B., Perlmann, P. and Wigzel, H. (eds) Lymphocytes. Isolation, fractionation and characterization. Scand. J. Immunol., Suppl. 5, Universitetsforlaget, Nyegaard,Oslo, pp. 244-267.

Boyum, M. A. (1974): Separation of blood leucocytes, granulocytes and lymphocytes. Tissue Antigens 4, 269-274.

Fritsche, K. L., Cassity, N. A. and Huang, S. C. (1991): Effect of dietary fats on the fatty acid composition of serum and immune tissues in chickens. Poultry Sci. 70, 1213-1222.

Fritsche, K. L., Alexander, D. W., Cassity, N. A. and Huang, S. C. (1993a): Maternally-supplied fish oil alters piglet immune cell fatty acid profile and eicosanoid production. Lipids $\mathbf{2 8}$, $677-682$.

Fritsche, K. L., Huang, S. C. and Cassity, N. A. (1993b): Enrichment of omega-3 fatty acids in suckling pigs by maternal dietary fish oil supplementation. J. Anim. Sci. 71, 1841-1847.

German, J. B., Xu, R., Walzem, R., Kinsela, J. E., Knuckles, B., Nakamura, N. and Yokoyama, W. H. (1996): Effect of dietary fats and barley fiber on total cholesterol and lipoprotein cholesterol distribution in plasma of hamsters. Nutr. Res. 16, 1239-1249.

Harris, W. S., Connor, W. E., Inkeles, S. B. and Illingworth, D. R. (1984): Dietary omega-3 fatty acids prevent carbohydrate-induced hypertriglyceridemia. Metabolism 33, 1016-1019.

Herold, P. M. and Kinsella, J. E. (1986): Fish oil consumption and decreased risk of cardiovascular disease: a comparison of findings from animal and human feeding trials. Am. J. Clin. Nutr. 43, 566-598.

Kaštel', R., Magic, D. and Vajda, V. (1992): A rapid method of gas chromatography for estimating the quantity of free fatty acids in plasma (in Slovak). In: Almanac 'Chemical Methods and their Significance for Health in Man.' Xth International Conference, Bratislava, 1.11.1992, pp. 8-10.

Lee, T. H., Hoover, R. L. and Williams, W. D. (1985): Effect of dietary eicosapentaenoic and docosahexaenoic acids on in vitro neurophil and monocyte leukotriene generation and neutrophil function. N. Engl. J. Med. 312, 1217-1224.

Lokaj, V. and Oburkova, P. (1975): Evaluation of tetrazolium-reductase activity of leukocytes (in Czech). Immunol. Zprav. VI, 42-44.

Morisaki, N., Shinomiya, M., Matsuoka, N., Satto, Y. and Kumagai, A. (1983): In vivo effects of cis$5,8,11,14,17-20: 5(n-3)$ and cis-4,7,10,13,16,19-22:6 (n-3) on serum lipoproteins, platelet aggregation and lipid metabolism in the aorta of rats. Tohoku J. Exp. Med. 141, 397-405.

Nestel, P. J., Connor, W. E., Reerdon, M. E., Connor, S., Wong, S. and Boston, R. (1984): Suppression by diets rich in fish oil of low density lipoprotein production in man. J. Clin. Invest. 74, 82-89.

Nestel, P. J. (1986): Fish oil attenuates the cholesterol induced rise in lipoprotein cholesterol. Am. J. Clin. Nutr. 43, 752-757.

Vaughn, D. M., Reinhart, G. A. and Swaim, S. F. (1994): Evaluation of dietary n-6 to n-3 fatty acid ratios on leukotriene B synthesis in dog skin and neutrophils. J. Vet. Intern. Med. 8, 188-198. 